\title{
Unusual decrease of the cosmic ray intensity in May 2019 on the background of the minima solar activity
}

\author{
Liudmila Trefilova, Pavel G. Kobelev ${ }^{\circledR}$, Anatoly V. Belov ${ }^{\circledR}$, Eugenia A. Eroshenko ${ }^{\circledR \dagger}$, \\ Anaid A. Melkumyan ${ }^{\circledR}$, Victoria A. Oleneva ${ }^{\circledR}$, Victor G. Yanke ${ }^{\circledR}$ \\ Correspondence \\ Pushkov Institute of Terrestrial Magnetism, lonosphere and Radio Wave Propagation(IZMIRAN), Moscow, Russia, \\ trefilova@izmiran.ru,kosmos061986@yandex.ru, abelov@izmiran.ru, erosh@izmiran.ru, \\ amelkumyan6@gmail.com,olene@izmiran.ru, yanke@izmiran.ru
}

\section{OPEN ACCESS}

This work is published under the Creative Commons Attribution 4.0 International license (CC BY 4.0) Please note that individual, appropriately marked parts of the work may be excluded from the license mentioned or mathe license other copyright conditions. th such third party matis. in such third party material is not under the Creative Commons license any copying, editing or public repro duction is only permitted with the prior consent of the respective copy right owner or on the basis of relevan legal authorization regulations.

\section{Keywords}

solar activity; Forbush decrease; $\mathrm{CME}$, cosmic ray variations

\begin{abstract}
In May 2019 there was a long and sloping decreasing of cosmic ray's intensity (up to $~ 4 \%$ ), which was observed on neutron monitors. Despite this was a small decreasing compared to quasi-eleven-period variation, it stands out well in 24 th cycle of solar activity.

According to LASCO/SOHO and STEREO-A data from spectrometer in different UHF bands and from coronograph, there was a series of CMEs which affected on modulation of cosmic rays by creating a series of Forbush decreasing, which didn't restore. This series was connected to two active regions on sun and began on April 30 from "reversed halo" CME. This CME didn't reach the earth, but led to significant additional modulation of cosmic rays, mostly on east side. Later there was a series of smaller CMEs on May 1-6, which also didn't reach the earth, but were gradually approaching to Earth. Recent CMEs on 8-9 and 12-13 created a normal Forbush decreasing. In May 2019, cosmic rays shown again, that they can collect information about distant objects of geliosphere and transmit it to Earth. The ground-level detectors sometimes can observe an interaction of interplanetary disturbances, which didn't reach the earth. East CMEs are especially effective, because they closing magnetic field lines beyond the orbit of earth and can interfere the restoring of cosmic ray's intensity.
\end{abstract}

\section{Introduction}

Since July 2018, the calmest period in the minimum solar activity of the 24th solar cycle has been observed. We can notice that the first half of May 2019 stands out sharply. From April 28 to May 17, against the background of a deep minimum of solar activity, a remarkable event took place. Small (up to $4 \%$ ), but prolonged decrease in the intensity of cosmic rays measured by neutron monitors ( $\sim 10$ days). This decrease is considered a relatively small change in intensity in General, but rather unusual and interesting for minimal solar activity. We can highlight it on the variations of cosmic rays at the Moscow station (figure 1) and on the time dependence of the zero harmonic A0 in figure 3 , which will be discussed in detail below. It can be seen that the recovery started on 12 May, but the situation changed on May 16 and a new decline began. The next recovery started on May 17, which also lasted for 10 days. The total duration of the event is about 25 days. Such a large time interval indicates that there were significant changes in the characteristics of the interplanetary environment almost on the scale of the semi-heliosphere. 


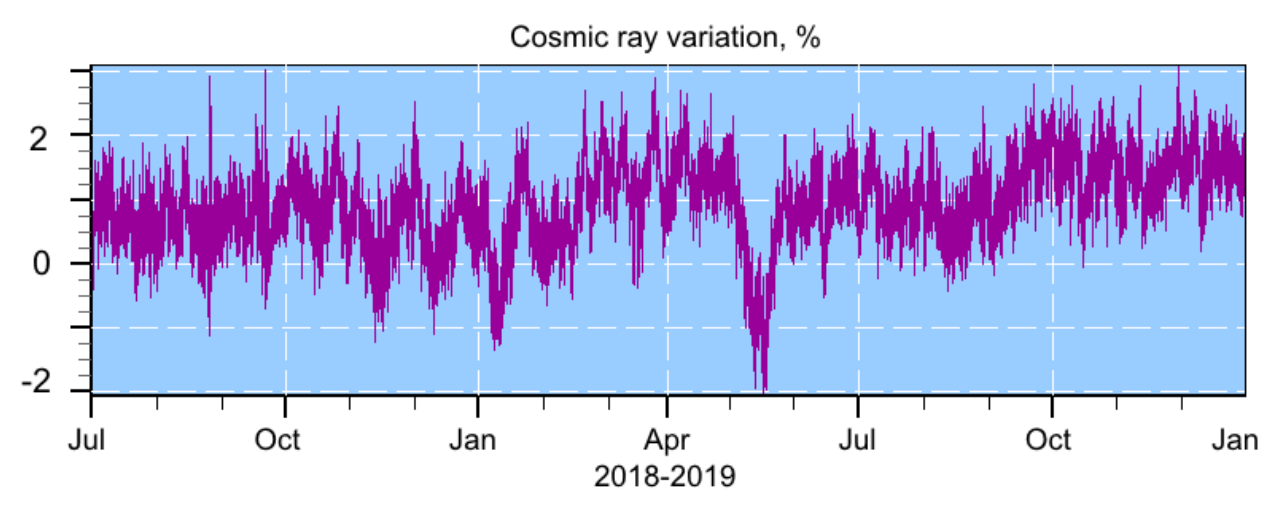

Figure 1: Hourly variations in the rate of counting cosmic rays according to the neutron supermonitor Moscow (July 2018-December 2019).

Such events are rare: a similar event was observed in September 1979 (Belov et al. 1983), but with a much greater decrease in the intensity of cosmic rays (more than $10 \%$ ). It was observed during the maximum of solar activity. This events are typical for such periods. A certain similarity with the event of May 2019 was also found in several other cases - in April 1980, July 2000 and July 2006 with amplitudes of $6 \%, 11 \%$ and $3.5 \%$, respectively. The first two were observed at the maximum of solar activity, the last one - at the end of the maximum- at the beginning of the minimum.

\section{Data analysis}

\subsection{Solar and geomagnetic conditions (activity) during the reviewingperiod}

According to SEEDS Monthly Catalog (http://spaceweather.gmu.edu/seeds/lasco.php, last accessed April 7, 2021), at this time (from April 28 to May 12), 13 significant CMEs were recorded (the angle of the ejection solution Angular Width $>50^{\circ}$, table 1) out of 38 observed during the eighteen-month quiet period (from July 2018 to December 2019), which is 35\%. Forbush effects observed near Earth's orbit in May 2019 according to the World network of neutron monitors, according to the "database of interplanetary perturbations and Forbush effects" (Belov et al. 2001). For each event, the maximum amplitude of the $\mathrm{FE}_{\text {mag }}$ effect, the maximum magnetic field and solar wind speed $\mathrm{B}_{\max }$ and $V_{\text {SWmax }}$, the $A x y_{\text {min }}$ projection of the cosmic ray anisotropy vector on the earth's Equatorial plane and the North-South projection $\mathrm{A}_{\mathrm{z}}$, and finally the rate of decreases $\mathrm{D}_{\min }$ are shown at the moment of the Forbush effect. All 13 emissions came from two regions that had been active for a long time. Constant emissions (including minor ones) did not allow the cosmic rays to recover and supported the process of slow decline.

\begin{tabular}{|l|ll|l|l|l|l|l|l|}
\hline & Date & Time & FEmag, $\%$ & $\mathrm{~B}_{\max } \mathrm{nT}$ & $\mathrm{V}_{\mathrm{SWmax}^{\prime}} \mathrm{km} / \mathrm{s}$ & $\mathrm{Axy}_{\min ^{\prime}} \%$ & $\mathrm{Az}, \%$ & $\mathrm{D}_{\text {min }^{\prime}} \% / \mathrm{h}$ \\
\hline $\mathbf{1}$ & $2019-05-01$ & $13: 00: 00$ & 0.9 & 9.1 & 569 & 1.08 & 0.81 & -0.26 \\
\hline $\mathbf{2}$ & $2019-05-03$ & $18: 00: 00$ & 0.6 & 9.8 & 505 & 0.87 & 1.16 & -0.28 \\
\hline $\mathbf{3}$ & $2019-05-07$ & $19: 00: 00$ & 0.8 & 11.9 & 380 & 0.96 & 1.21 & -0.20 \\
\hline $\mathbf{4}$ & $2019-05-09$ & $06: 00: 00$ & 0.5 & 11.9 & 380 & 0.64 & 1.18 & -0.21 \\
\hline
\end{tabular}




\begin{tabular}{|l|ll|l|l|l|l|l|l|}
\hline $\mathbf{5}$ & $2019-05-10$ & $18: 00: 00$ & 1.3 & 14.3 & 556 & 0.99 & 0.75 & -0.33 \\
\hline $\mathbf{6}$ & $2019-05-14 \quad 00: 00: 00$ & 1.1 & 14.3 & 556 & 1.34 & 1.07 & -0.35 \\
\hline $\mathbf{7}$ & $2019-05-16 \quad 00: 00: 00$ & 0.9 & 8.4 & 480 & 1.08 & 1.13 & -0.28 \\
\hline
\end{tabular}

Table 1: Forbush effects observed near Earth's orbit in May 2019.

Forbush decrease is a short-term and drastic decrease in the intensity of galactic cosmic rays. The effect is explained by the increased scattering of galactic cosmic rays by perturbations of the interplanetary magnetic field carried by the solar wind from the Sun to the borders of the heliosphere.

The strongest CMEs from these active areas were observed from April 28 to May 12, 2019. On April 28, an ejection occurred beyond the Western limb. However, this ejection did not alone create such a decrease, at this time also came the disturbance created by the flow of plasma from the coronal hole observed in the center of the solar disk on April 27. Then there was a strong release on April 30 (figure 2, source: https://sohowww.nascom.nasa.gov/explore/coronagraph.html, last accessed June 4, 2021)"

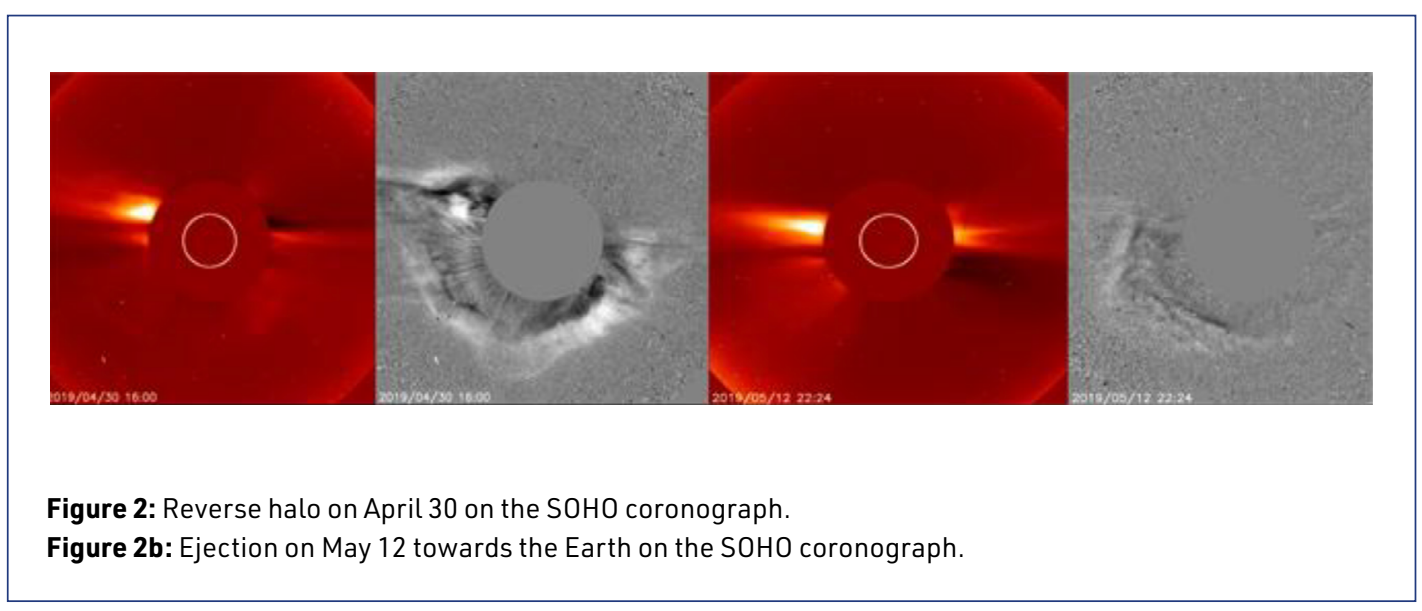

On the chronograph, it was a reverse halo. The next ejection was observed for the Eastern limb on May 1. Further, from May 3 to May 5, a number of ejections were observed on the Eastern limb, which gave Forbush declines on May 6, 7 and 8. But on May 6, there was an outburst in the East of the visible solar disk, and on May 9, there was a slight Forbush decrease. On May 8 and 9, there were ejections in the visible Eastern part of the solar disk, giving a number of Forbush decreases on May 10 and 11. Such continuous CMEs did not allow the cosmic rays to recover. It was only after May 12 that the recovery started, as no significant processes were observed for several days. However, on May 12, there was again a fairly large ejection in the form of a direct halo (figure 2b), which led to a Forbush decrease on May 16, without allowing the cosmic rays to fully recover. No events were observed after May 13, and cosmic rays began to recover from May 17 and fully recovered by May 26 . 


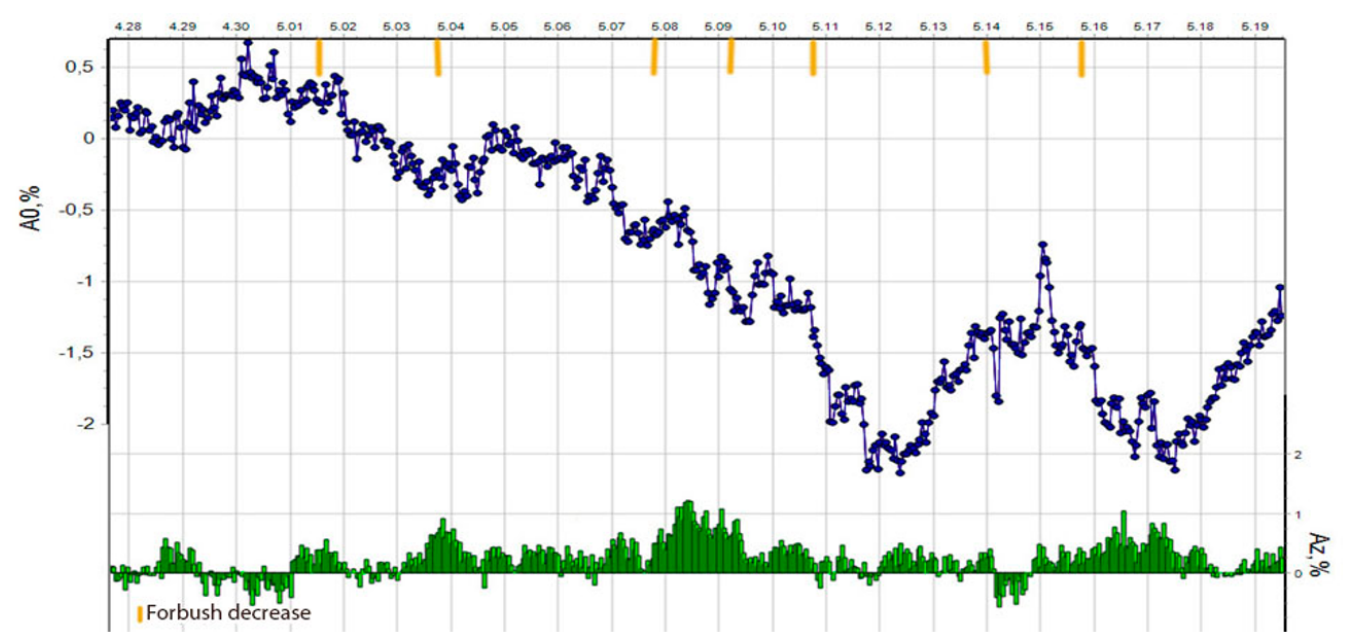

Figure 3: The Amplitude of the zero harmonic A 0 for an uncharacteristic decrease in the rate of counting cosmic rays in May 2019

\subsection{Method of selecting Forbush effects for the May decrease}

Here is an example for the events of May 1, where the maximum amplitude of the $\mathrm{FE}_{\text {mag }}$ effect reached $0.9 \%$. Each Forbush was selected by changes in various parameters, mainly components of the magnetic field, solar wind speed, density, and temperature. Figure 4 shows a significant increase in wind speed, and the other parameters mentioned above have also increased slightly.

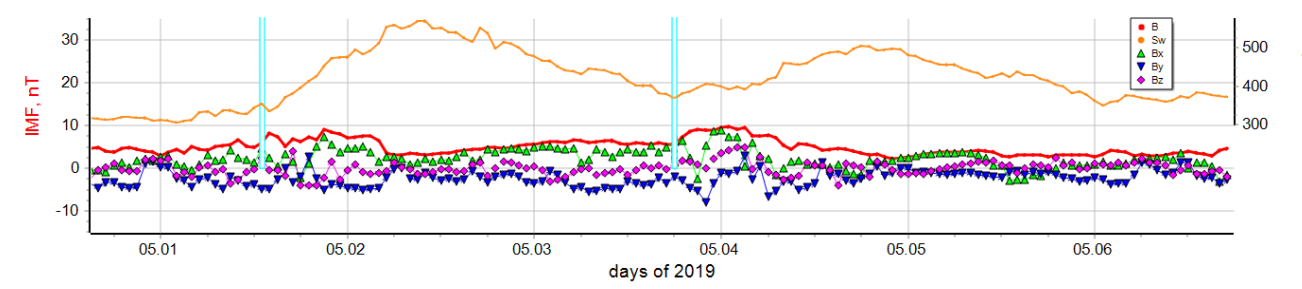

Figure 4: Solar wind parameters.

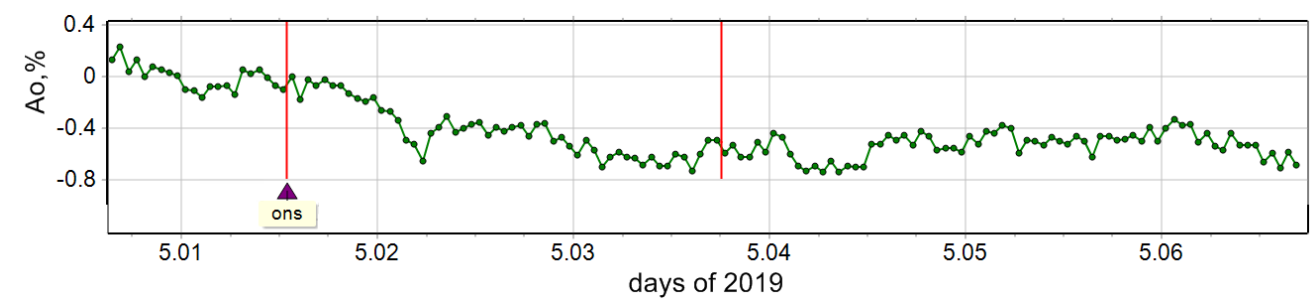

Figure 5: The amplitude of the zero harmonic A0 for an uncharacteristic decrease in the speed of counting cosmic rays in May 2019. 
Then there is some decrease in all indicators, which is replaced by another growth, which indicates the next Forbush decrease. Thus, we get a Forbush decrease with subsequent recovery (figure 4). By transferring the time interval to the graph of the zero harmonic amplitude (figure 5), we can see that there is a pre-reduction of the Forbush effect in this event. The same method is used for all other events.

Analysis of the characteristics of the solar wind in General allows us to see that due to the significant frequency of flares that followed the initial decrease due to the activity of coronal holes on May 1-3, and then due to emissions from active regions, cosmic rays did not have time to recover, forming a number of Forbush effects, which led to a long total decrease.

\subsection{Results of the analysis of the zero harmonic of cosmic rays}

Analyzing the data of neutron monitors with an effective energy of $10 \mathrm{GeV}$, we can distinguish a number of individual decreases separated by sections of elevation or plateau (figure 3). These slopes are caused by a lot of small Forbush decreases following each other, up to $1.5 \%$. Forbush effects are considered significant from $3.5 \%$ at a proton energy of 10 . However, after such small decreases, there is no recovery for a long time.

\subsection{Distribution of solar wind density for CMEs in April-May}

Based on the solar wind density model presented on the website http://helioweather.net/archive/2019/05/den2e4-earth_201905.mp4 (last accessed April 23, 2021), we can see its detailed distribution during outliers.

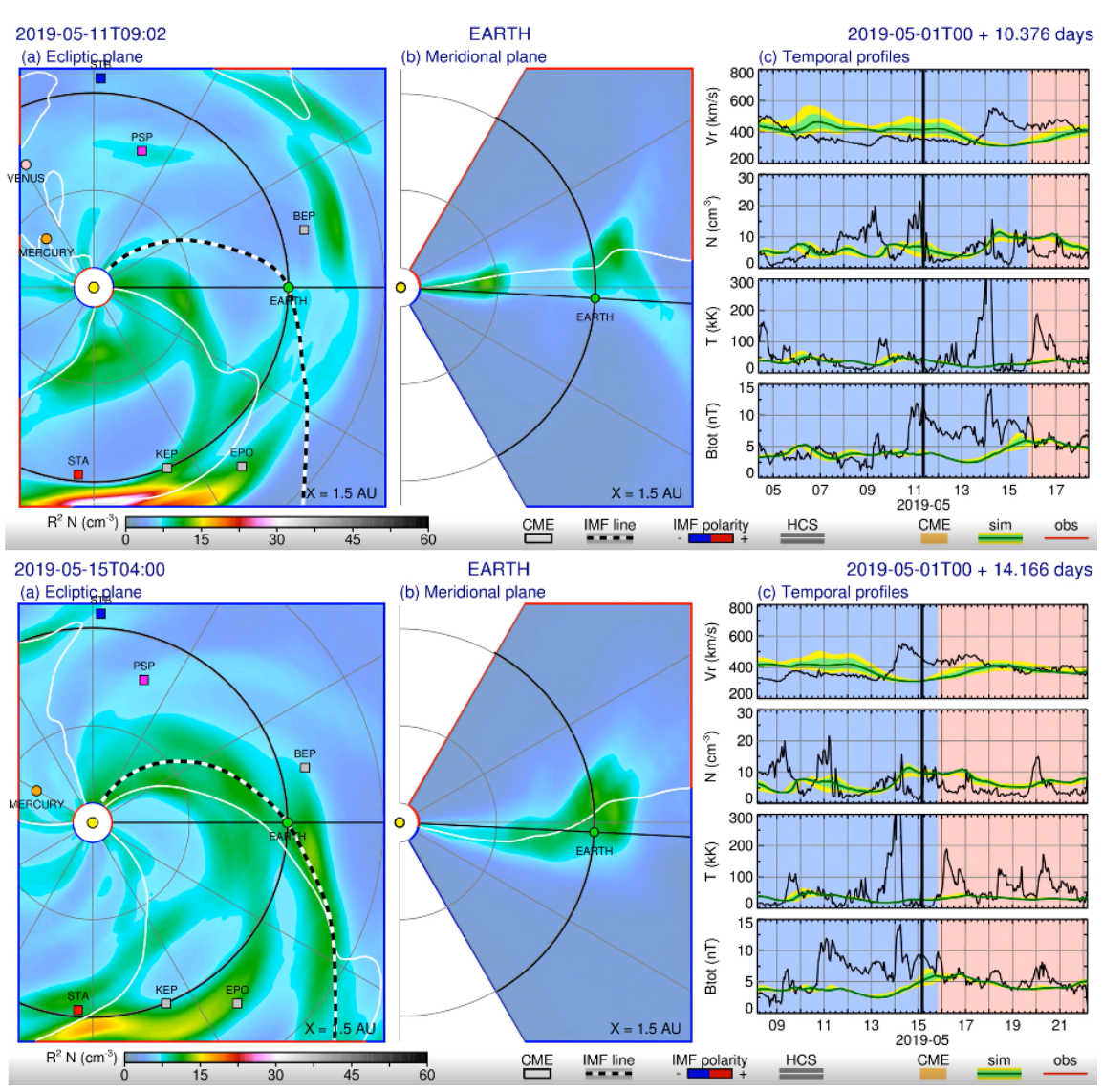

Figure 6: Behavior of solar wind density on May 11 (left), solar wind density behavior on May 15 (right). 


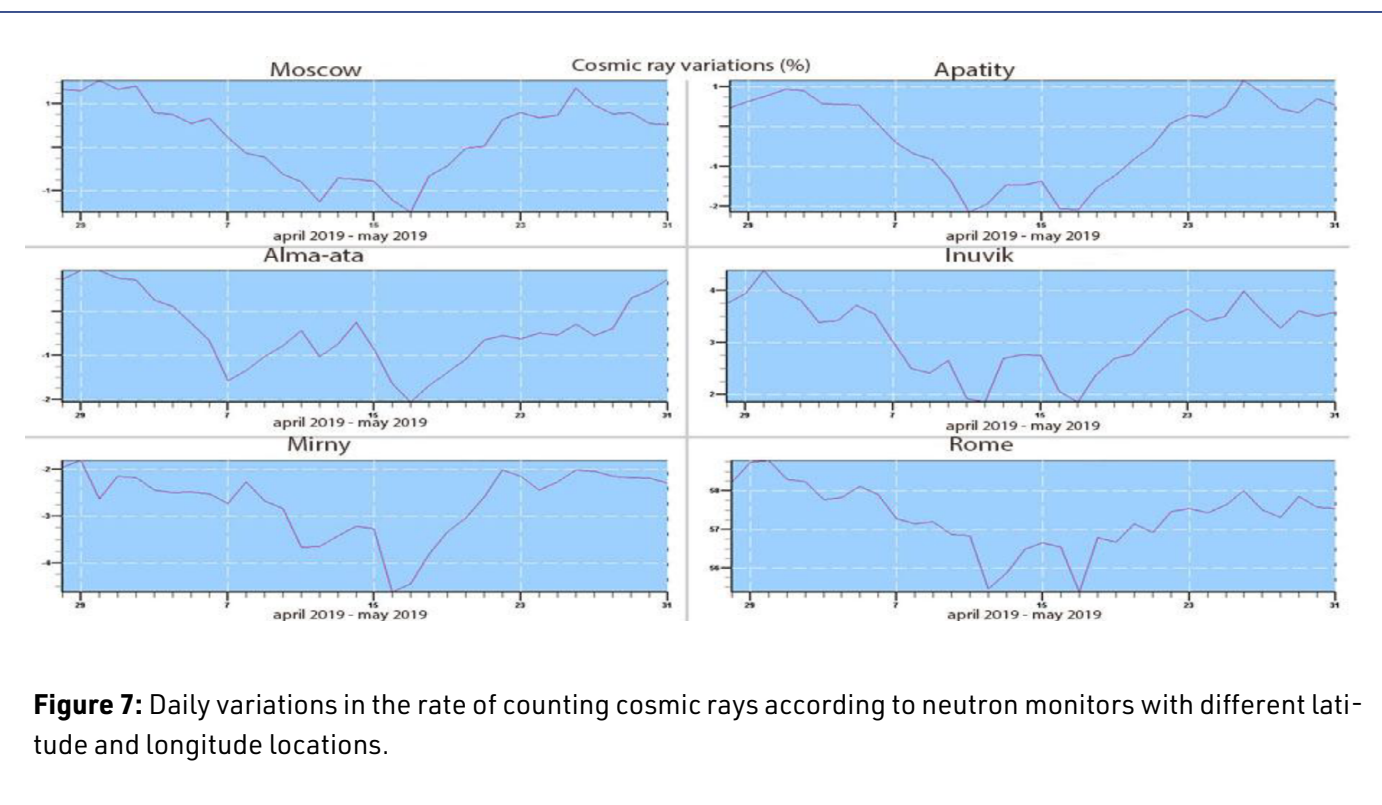

Inside the CMEs, the solar wind density reaches much higher values than usual. High-density plasma closes the lines of force for cosmic rays in certain directions, thus reducing the number of detected particles. Figure 6a shows the ejections that occurred on April 8 behind the Eastern limb, as a result-the decrease in cosmic rays on May 11. Despite the fact that the ejections do not reach the Earth, they influenced the count rate of neutron monitors. Figure $6 \mathrm{~b}$ shows the distribution of density from direct CMEs towards the Earth on May 12 and 13, which led to a decrease in cosmic rays on May 16-17. This minimum intensity is visible on all monitors (figure 7).

The flow of cosmic rays is strongly influenced by magnetic fields. Under normal conditions, the magnetic field can be represented as a quasi-spiral, cosmic rays move along lines of force, mostly in the direction of the sun. The ejection can be represented as a ring with a field wound on it, which has different values and is directed mostly across the magnetic lines of the sun. The lines of force are moving behind the shock wave from the release. Transverse lines of force create a kind of screen for cosmic rays, that have been involved in a gradually expanding flow cannot overcome the cross-flow. In these areas, the exchange of cosmic rays is difficult, and recovery is worse. However, cosmic rays, not being able to pass through the force line, will seek to find another path. This reduces the recovery flow compared to normal, which leads to a decrease in the intensity of cosmic rays, which is an important condition for the appearance of Forbush decreases.

\subsection{The decreasing on the other planets}

Looking at the location of the planets relative to the Sun in the beginning of May, we can say that dedicated emissions in considered period could influence as well as on Mars and Jupiter. We can conclude it looking at the date on figure. It could be said that investigated phenomenon has global character. There are cosmic ray variations on the Earth, Mars, and electrons from Jupiter, recorded near the Earth presented in figure 8.

A detailed description of the RAD instrument can be found in Hassler et al. (2012) and data from base (RAD, 2020). Since Mars was opposite the eastern limb, as a result of the powerful solar event April 28 in early May on the RAD detector on Mars, the probability of a significant Forbush decrease was higher than that of Earth. However, on the Martian detector, the variations had, on the whole, the same time dependence and approximately the same magnitude as near the Earth for $10 \mathrm{GV}$. However, on May 9-10 and 21-22, Forbush decreases were observed at the RAD detector, with a value of $2-3 \%$, which indicates the global nature of the phenomena under study. 


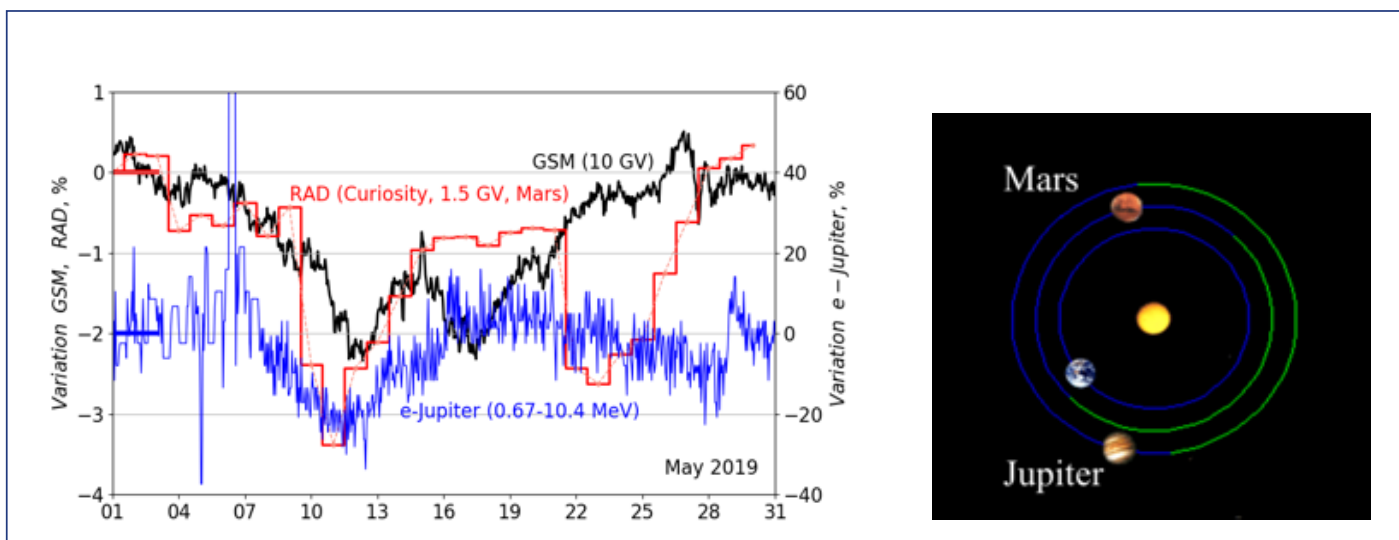

Figure 8: Cosmic ray variations on the Earth, Mars and electrons from Jupiter, recorded near the Earth.

A detailed description of the SOHO/EPHIN instrument can be found in Müller-Mellin et al. (1995) and data from base (EPHIN 2020). The variations in the electron flux of Jupiter near the Earth's orbit are an order of magnitude larger than the variations in the flux of protons $10 \mathrm{GV}$, and the time course repeats in the first half of May. But, as follows from figure 8 Earth and Jupiter were not on the same line of force at that moment. And this also testifies in favor of a global decrease in the CR intensity.

\section{Discussion and conclusion}

The hypothesis of series of small events followed each other was proved by analysis of global survey method, which allows us to highlight separate events from the series.

There was a period of splash during the minimum of Solar activity, that was great enough. Perhaps, we could observe one of the greatest Forbush effect in the cycle, when CME on April 30 had been directed to the Earth.

We can notice the influence of emissions on the Earth, which are not able to come there, special for eastern. Cosmic rays come from huge area therefore we can see more eastern events or even events out of nthe limb by neutron monitor data. The modulation of cosmic rays is depending on situation in whole heliosphere. There is fundamental difference between cosmic ray variations and other geophysical effects.

\section{Acknowledgments}

This work was partially supported by the grant RFBR No 18-02-00451. Experimentally and methodologically support the project USU Russian national network of ground stations of cosmic rays. We are grateful to all the staff of the World Network of cosmic ray stations http://cr0.izmiran.ru/ ThankYou.

RAD is supported by NASA (HEOMD) under JPL subcontract \#1273039 to Southwest Research Institute and in Germany by DLR and DLR's Space Administration grant numbers 50QM0501, 50QM1201, and 50QM1701 to the Kiel University.

The SOHO/EPHIN project is supported under grant $500 \mathrm{C} 1702$ by the German Bundesministerium für Wirtschaft through the Deutsches Zentrum für Luft- und Raumfahrt (DLR).

We acknowledge the NMDB (https://www.nmdb.eu/), founded under the European Union's FP7 programe (contract no. 213007) for providing the data. 


\section{References}

Abunina, M., Abunin, A.A., Belov, A.V., Eroshenko, E.A., Asipenka, A.S., Oleneva, V.A., Yanke, V.G., 2018, Relationship between the parameters of Forbush effects and heliolongitude of solar sources Geomagnetism and aeronomy, V.51, 3, P.374-389, D0I: https://dx.doi. org/10.7868/s0016794018030082

Belov, A.V., Eroshenko, E.A., Yanke, V.G., Oleneva, V.A., Abunina, M., Abunin, A., 2018, Global survey method for the world network of neutron monitors, Geomagnetism and aeronomy, V.58, 3, P.374-389, DOI: https://dx.doi.org/10.7868/s0016794018030082

Belov, A.V., 2008, Forbush effects and their connection with solar, interplanetary and geomagnetic phenomena September, Proceedings of the International Astronomical Union 4(S257):439-450, DOI: https://dx.doi.org/10.1017/S1743921309029676

Belov, A.V., Dorman, L.I., Eroshenko, E.A., Melkumyan, A.A., 1983 , "Two great cosmic ray intensity decreases in August and September 1979", Cosmic Rays (Moscow, NAUKA), Vol. 23, 60-63

Belov, A. V., Eroshenko, E. A., Oleneva, V. A., Yanke, V. G., Mavromichalaki, H., 2006, Long-term variations of the cosmic ray anisotropy by the data from neutron monitor network, Proc. 20th ECRS, Lisbon, 123

Hassler, D., Zeitlin, C., Wimmer-Schweingruber, et al., 2012, The Radiation Assessment Detector (RAD) investigation. Space Sci. Rev. 170 (1-4), 503, DOI: https://dx.doi.org/10.1007/s11214-012-9913-1

Müller-Mellin, R., Kunow, H., Fleißner, V., et al., 1995, COSTEP-Comprehensive Suprathermal and Energetic Particle Analyser, Solar Physics, V.162, P.483-504, DOI: https://dx.doi.org/10.1007/BF00733437

RAD, 2020, RAD data can be found in NASA's PDS or at the RAD web page https://atmos.nmsu.edu/data_and services/atmospheres data/ INSIGHT/insight.html (last accessed April 7, 2021)

EPHIN, 2020. The EPHIN data used in this study as well as a detailed documentation of the new data product can be found at http://ulysses. physik.uni-kiel.de/costep/level2/rl2 and in the SOHO archive at https://soho.nascom.nasa.gov/data/archive (last accessed April 7, 2021)

\section{Questions and answers}

Monica Laurenza: Did you associate an ICME counterpart for each FD?

Answer: Yes, of course. In most cases, this relationship was clearly defined.

Rolf Bütikofer: How do you explain the very short decrease on14 May 2019?

Answer: The 14 May 2019 decrease is also a Forbush effect that recovered very quickly. This is due to the Western position of the solar source.

Ludwig Klein: Why are the recoveries of these (and other) FDs rather fast, while those of large FDs are very gradual (layman's question)?

Answer: The important is not the recovery time, but the recovery speed, which is usually not very different in cases of large and small Forbush decrease. 